\section{Fatores associados ao deslocamento ativo em escolares}

\section{Associated factors to children's active commuting to school}

\author{
Ricardo Rodrigo Rech ${ }^{1,2,3}$ \\ Caroline Origotti da Rosa ${ }^{4}$ \\ Paulo Roberto Avrela ${ }^{4}$ \\ Ricardo Halpern ${ }^{1,5}$ \\ Cristine Boone Costanzi ${ }^{4}$ \\ Mauren Lucia de Araujo Bergmann ${ }^{7}$ \\ Lidiane Requia Alli ${ }^{6}$ \\ Josué Luís Pedroni ${ }^{1}$
}

\section{Resumo}

Objetivou-se verificar o tipo de deslocamento à escola em estudantes de 7 a 12 anos na cidade de Caxias do Sul. Participaram 1442 escolares que responderam a um questionário sobre o modo de deslocamento adotado para o trajeto casa/escola. As variáveis independentes foram idade, sexo, deslocamento ativo à escola, classificação econômica, escolaridade materna, tipo de escola e quantidade de carros na residência. Os resultados mostraram que 58,1\% dos escolares se deslocam ativamente, onde os meninos apresentaram um percentual maior do que as meninas, porém, sem diferença estatística significante. Os estudantes mais velhos apresentaram 63\% mais chances de se deslocarem ativamente do que os mais novos. Os escolares com condição socioeconômica mais favorável apresentaram mais do que o dobro de chances de não se deslocarem ativamente do que seus pares. Estes dados mostram que serão necessárias diversas intervenções para melhorar a promoção da saúde e qualidade de vida dos escolares.

\section{Palavras-chave}

Deslocamento; Escolares; Atividade física.

\begin{abstract}
This study aimed to verify the way to commute to school on 7-12 years students in the city of Caxias do Sul. 1442 students participated and answered a questionnaire on the commuting adopted for the homel school route. The independent variables were age, gender, active commuting to school, economic status, maternal education, type of school and number of cars in the residence. The results showed that $58.1 \%$ of the students move actively, where the boys had a higher percentage than girls, however, not statistically significant. Older students were 63\% more likely to move actively than the younger ones. Students with more favorable socioeconomic conditions were more than twice as likely not to move actively than their peers. These data show that different interventions are needed to improve health promotion and life quality of schoolchildren.
\end{abstract}

\section{Keywords}

Commuting; School children; Physical activity.
Rev Bras Ativ Fis Saúde p. 332-338 DOI:

http://dx.doi.org/10.12820/rbafs.v.18n3p332

1 Programa de Pós-Graduação em Ciências da Saúde, Universidade Federal de Ciências da Saúde de Porto Alegre, Porto Alegre, RS.

2 Núcleo de Pesquisa Ciências e Artes do Movimento Humano. Universide de Caxias do Sul, Caxias do Sul, RS.

3 Núcleo de Pesquisa em Saúde Coletiva. Universide de Caxias do Sul, Caxias do Sul, RS.

4 Universidade de Caxias do Sul, Caxias do Sul, RS.

5 Programa de Pós-Graduação em Saúde Coletiva, Universidade Luterana do Brasil, Canoas, RS.

6 Universidade Luterana do Brasil, Canoas, RS. 7 Universidade Federal do Pampa, Uruguaiana, $R S$. 


\section{INTRODUÇÃO}

O sedentarismo, em crianças e adolescentes, é considerado um problema de saúde pública devido à sua associação com a obesidade na infância e maior morbidade na idade adulta. ${ }^{1} \mathrm{~A}$ aptidão física para crianças e adolescentes deve ser desenvolvida como primeiro objetivo de incentivo a adoção de um estilo de vida apropriado com a prática de exercícios por toda a vida, com o intuito de desenvolver e manter um condicionamento físico suficiente para melhoria da capacidade funcional e da saúde. ${ }^{2}$ Os benefícios à saúde podem ser provenientes da prática de atividades físicas em níveis recomendados, que nos jovens englobam a realização de atividades físicas de intensidade moderada a vigorosa por no mínimo $60 \mathrm{mi}-$ nutos e que sejam agradáveis e adequadas para o crescimento e desenvolvimento dos mesmos. ${ }^{3}$

Ainda que a utilização de modos de transporte fisicamente ativos influenciem positivamente na atividade física habitual dos jovens, a proporção de crianças e adolescentes que caminham ou vão de bicicleta para a escola ainda é pequena. No Brasil, ainda há poucos levantamentos abordando a prática de atividade física dos sujeitos em seus deslocamentos. Os estudos que focalizaram esta temática identificaram que $20 \%$ a $30 \%$ dos jovens parecem estar expostos à inatividade física nos deslocamentos. ${ }^{4}$

Apesar das evidências mostrarem que mudanças em simples hábitos diários (como caminhar de casa até a escola), influenciam positivamente nos hábitos de vida das crianças, é comum vermos um elevado número de pais levarem os filhos à escola de automóvel..$^{5}$ Diante do exposto, o estudo buscou verificar qual o tipo de deslocamento à escola em estudantes de 7 a 12 anos na cidade de Caxias do Sul, RS.

\section{MÉTODOS}

Trata-se de um estudo epidemiológico transversal de base escolar. Os critérios de amostragem e a logística do estudo já foram descritos em Rech et $\mathrm{al}^{6}$.

Foram utilizados questionários para avaliação das variáveis sexo, idade, tipo de moradia, nível socioeconômico e tipo de deslocamento para a escola (vai para a escola caminhando, pedalando ou outro meio de transporte). Estas variáveis foram obtidas através de entrevista e testados em estudo piloto.

Informações referentes a classe socioeconômica foram avaliadas utilizando-se a classificação de Barros e Victora ${ }^{7}$ que considera a utilização de 13 variáveis para produzir o indicador econômico nacional (IEN).

Quanto aos aspectos éticos, todos os escolares avaliados receberam e retornaram assinado o termo de consentimento livre e esclarecido (TCLE) assinado pelos pais ou responsáveis além de serem voluntários para o estudo. $\mathrm{O}$ estudo foi aprovado pelo Comitê de Ética em Pesquisa (CEP) da Universidade Luterana do Brasil com número de protocolo 2006-365H.

Foi utilizada estatística descritiva (frequências e percentuais) e análise bivariada (teste qui-quadrado de Pearson) entre as variáveis independentes e o desfecho. O nível de significância adotado foi de $95 \%$. 


\section{RESULTADOS}

Das 1573 crianças selecionadas para o estudo, 1442 foram avaliadas. Trinta crianças se recusaram a participar do estudo e 38 não apresentaram o TCLE. Foram excluídos 45 questionários que apresentaram inconsistência de dados. Também foram excluídas da análise 11 crianças que estavam fora da faixa etária estipulada para o estudo. As perdas e recusas somaram $8,4 \%(\mathrm{n}=131)$.

A média de idade dos avaliados foi de 9,59 anos ( $\mathrm{DP}=1,54)$. As demais características da amostra estão descritas na tabela 1 . A prevalência de deslocamento ativo à escola foi de 58,1\% ( $\mathrm{n}=830)$.

Tabela 1 - Características da amostra*

\begin{tabular}{|c|c|c|}
\hline & $n$ & $\%$ \\
\hline \multicolumn{3}{|l|}{ Idade } \\
\hline 7 & 149 & 10,3 \\
\hline 8 & 261 & 18,1 \\
\hline 9 & 276 & 19,1 \\
\hline 10 & 295 & 20,5 \\
\hline 11 & 274 & 19,0 \\
\hline 12 & 187 & 13,0 \\
\hline \multicolumn{3}{|l|}{ Sexo } \\
\hline Meninos & 721 & 50,0 \\
\hline Meninas & 721 & 50,0 \\
\hline \multicolumn{3}{|l|}{ Deslocamento ativo à escola } \\
\hline Sim & 830 & 58,1 \\
\hline Não & 599 & 41,9 \\
\hline \multicolumn{3}{|l|}{ Classificação econômica } \\
\hline Classes Baixa e Intermediária & 481 & 41,0 \\
\hline Classe Alta & 691 & 59,0 \\
\hline \multicolumn{3}{|l|}{ Escolaridade Materna } \\
\hline Até ensino fundamental & 651 & 55,1 \\
\hline Ensino médio ou superior & 530 & 44,9 \\
\hline \multicolumn{3}{|l|}{ Tipo de Escola } \\
\hline Privada & 199 & 13,8 \\
\hline Pública & 1243 & 86,2 \\
\hline \multicolumn{3}{|c|}{ Quantidade de carros na residência } \\
\hline nenhum ou 1 carro & 1243 & 86,9 \\
\hline dois ou mais carros & 187 & 13,1 \\
\hline
\end{tabular}

* devido a algumas respostas em branco, em alguns campos o $n$ total é inferior a 1442.

A tabela 2 apresenta os resultados da análise bivariada entre deslocamento ativo e as variáveis independentes. Meninos e meninas não apresentaram diferença significante no deslocamento ativo a escola $(p>0,05)$. Todas as demais variáveis apresentaram associação estatística significante com o desfecho. 
Tabela 2 - Análise bivariada entre deslocamento ativo à escola e variáveis independentes

\begin{tabular}{|c|c|c|c|c|c|}
\hline \multicolumn{6}{|c|}{ Deslocamento ativo à escola } \\
\hline & & Sim & Não & $\mathrm{RP}$ & IC \\
\hline \multicolumn{6}{|l|}{ Idade } \\
\hline \multirow[t]{2}{*}{ 07, 08 e 09 anos } & $\mathrm{n}$ & 354 & 323 & \multirow[t]{2}{*}{1,00} & \\
\hline & $\%$ & $52,3 \%$ & $47,7 \%$ & & \\
\hline \multirow[t]{2}{*}{10,11 e 12 anos } & $\mathrm{n}$ & 476 & 276 & \multirow[t]{2}{*}{$0,63 *$} & \multirow[t]{2}{*}{$0,51-0,78$} \\
\hline & $\%$ & $63,3 \%$ & $36,7 \%$ & & \\
\hline \multicolumn{6}{|l|}{ Sexo } \\
\hline Feminino & $\mathrm{n}$ & 407 & 310 & \multirow[t]{2}{*}{1,00} & \\
\hline & $\%$ & $56,8 \%$ & $43,2 \%$ & & \\
\hline \multirow[t]{2}{*}{ Masculino } & $\mathrm{n}$ & 423 & 289 & \multirow[t]{2}{*}{0,90} & \multirow[t]{2}{*}{$0,73-1,11$} \\
\hline & $\%$ & $59,4 \%$ & $40,6 \%$ & & \\
\hline \multicolumn{6}{|l|}{ Classificação econômica } \\
\hline \multirow[t]{2}{*}{ Baixa e Intermediária } & $\mathrm{n}$ & 343 & 137 & \multirow[t]{2}{*}{1,00} & \\
\hline & $\%$ & $71,5 \%$ & $28,5 \%$ & & \\
\hline \multirow[t]{2}{*}{ Classe Alta } & $\mathrm{n}$ & 338 & 352 & \multirow[t]{2}{*}{$2,61^{*}$} & \multirow[t]{2}{*}{$2,03-3,34$} \\
\hline & $\%$ & $49,0 \%$ & $51,0 \%$ & & \\
\hline \multicolumn{6}{|l|}{ Escolaridade Materna } \\
\hline Até ensino fundamental & $\mathrm{n}$ & 431 & 219 & \multirow[t]{2}{*}{1,00} & \\
\hline & $\%$ & $66,3 \%$ & $33,7 \%$ & & \\
\hline \multirow[t]{2}{*}{ Ensino médio ou superior } & $\mathrm{n}$ & 251 & 276 & \multirow[t]{2}{*}{$2,16^{*}$} & \multirow[t]{2}{*}{$1,71-2,74$} \\
\hline & $\%$ & $47,6 \%$ & $52,4 \%$ & & \\
\hline \multicolumn{6}{|l|}{ Tipo de Escola } \\
\hline \multirow[t]{2}{*}{ Privada } & $\mathrm{n}$ & 15 & 182 & \multirow[t]{2}{*}{1,00} & \\
\hline & $\%$ & $7,6 \%$ & $92,4 \%$ & & \\
\hline \multirow[t]{2}{*}{ Pública } & $\mathrm{n}$ & 815 & 417 & \multirow[t]{2}{*}{$0,04^{*}$} & \multirow[t]{2}{*}{$0,02-0,07$} \\
\hline & $\%$ & $66,2 \%$ & $33,8 \%$ & & \\
\hline \multicolumn{6}{|l|}{ Num. carros na residência } \\
\hline nenhum ou 1 carro & $\mathrm{n}$ & 764 & 476 & 1,00 & \\
\hline & $\%$ & $61,6 \%$ & $38,4 \%$ & & \\
\hline dois ou mais carros & $\mathrm{n}$ & 65 & 121 & $209 *$ & 112 \\
\hline & $\%$ & $34,9 \%$ & $65,1 \%$ & $2,99^{*}$ & $2,16-4,12$ \\
\hline
\end{tabular}

RP = Razão de Prevalências; IC = Intervalo de Confiança; * $\mathrm{p}<0,05$.

\section{DISCUSSÃO}

O presente estudo encontrou $58,1 \%$ de escolares que se deslocam ativamente à escola. Em adolescentes dinamarqueses as seguintes prevalências de deslocamento à escola foram observadas: $41,6 \%$ a pé, $33,0 \%$ por meio de bicicleta, $14,3 \%$ de ônibus, $10,3 \%$ de carro e $2,6 \%$ por motocicleta. ${ }^{8}$ Estudo realizado com adolescentes espanhóis apresentou prevalência de deslocamento ativo para a escola de 65\%. ${ }^{9} \mathrm{Na}$ Irlanda, 36,4\% das crianças se deslocam ativamente para a escola. ${ }^{10} \mathrm{Em}$ escolares da cidade de João Pessoa, Paraíba, com idade de sete a 12 anos, foi constatada a prevalência de 70,0\% de deslocamento ativo. ${ }^{11} \mathrm{Na}$ cidade de Pelotas-RS, Hallal et $\mathrm{al}^{12}$ observaram prevalência de deslocamento ativo à escola de 72,8\% e Borfe et $\mathrm{al}^{5}$ em Santa Cruz do Sul-RS encontraram 54,8\% de escolares que se deslocavam ativamente à escola. Pode-se dizer que nos países desenvolvidos o deslocamento ativo à escola torna-se mais favorável, principalmente pela melhor condição das vias públicas. ${ }^{13}$ Porém os resultados apresentados mostram um percentual considerável de escolares se deslocando ativamente na cidade avaliada. Outro fator relevante que pode ser considerado pelos pais como uma barreira para o deslocamento ativo é a distância entre a escola e a casa ${ }^{14}$, onde crianças que 
vivem mais próximas da escola apresentam maiores chances de se deslocarem de maneira ativa. ${ }^{15}$

Meninos e meninas não apresentaram diferença estatística significante em relação ao deslocamento ativo, porém os meninos apresentaram um percentual superior de deslocamento ativo à escola em relação às meninas. Estudo realizado na Irlanda relatou que os meninos foram significativamente mais ativos do que as meninas. ${ }^{10}$ No estudo de Itabuna-BA, os meninos também apresentaram maior nível de deslocamento ativo à escola. ${ }^{13} \mathrm{Na}$ cidade de Pelotas-RS, os resultados apresentaram o sexo masculino mais ativo do que feminino. ${ }^{12}$ Em contra partida, em um estudo com escolares finlandeses observou que as moças apresentaram maior proporção de deslocamento ativo à escola do que os rapazes. ${ }^{16} \mathrm{O}$ sexo pode determinar diferenças nos padrões de atividade física de crianças e adolescentes ${ }^{17}$ e estas diferenças na adoção de hábitos ou práticas podem ser em parte explicadas por fatores culturais, comportamentais, psicológicos e até mesmo maturacionais que afetam esta faixa etária. ${ }^{18}$

Os escolares mais velhos apresentaram 37\% menos chances de não se deslocarem ativamente em relação aos mais novos. Com adolescentes Espanhóis, Chillon et $\mathrm{al}^{9}$ observaram um declínio na realização de deslocamento ativo à escola com o aumento da idade. $\mathrm{Na}$ cidade de Toronto, Canadá, a prevalência de deslocamento ativo para a escola (de 1986 a 2001) diminuiu de 53\% para 42\% nas idades de 11-13 anos e de 39\% para 31\% para as idades de $14-15$ anos. ${ }^{19} \mathrm{Um}$ fator que pode explicar em parte tais resultados é a possibilidade de uma maior tendência à permissão dos adolescentes mais velhos a realização de deslocamentos ativos, contudo, vale salientar que essa maior tendência ocorre em adolescentes provenientes de escolas públicas. ${ }^{12,11}$

Os escolares de condição econômica mais favorável apresentaram mais do que o dobro de chances de não se deslocarem ativamente do que aqueles com piores condições. Em um estudo com crianças e adolescentes canadenses foi observado que a baixa renda é um dos fatores preditivos para o deslocamento ativo. ${ }^{20} \mathrm{Hallal}$ et $\mathrm{a}^{12}$ observaram que o deslocamento a pé associou-se negativamente com o nível socioeconômico, sendo o percentual de caminhada entre os adolescentes do nível E seis vezes maior do que o observado no nível A. A utilização de carro ou moto mostrou tendência contrária, sendo que os adolescentes do nível A utilizaram carro ou moto como modo de transporte 63 vezes mais do que os do nível E. Esse resultado é provavelmente explicado pela ausência de uma alternativa de transporte, ao invés de real consciência da população sobre os benefícios do deslocamento ativo. Também se pode citar o fato que algumas famílias de maior renda podem contratar cuidadores para conduzir seus filhos da escola para casa, uma opção que pode ser financeiramente indisponível para famílias de baixa renda. Outro fator que também pode interferir nesse aspecto é a programação dos pais, ou seja, alguns pais levam seus filhos à escola no período da manhã, quando vão trabalhar, porém, quando seus filhos deixam a escola no período da tarde, eles ainda estão trabalhando. ${ }^{21}$

O presente estudo também encontrou que os estudantes de escola pública apresentaram 96\% menos chances de não se deslocarem ativamente do que os de escola privada. Em João Pessoa-PB, também foi encontrado que escolares da rede privada se deslocam, em sua maioria, passivamente para a escola $(59,4 \%)$ enquanto que os da rede pública apresentaram percentual menor de deslocamento passivo (16,6\%). ${ }^{11} \mathrm{Na}$ cidade de Santa Cruz do Sul-RS, dos escolares que estudam em escola pública na periferia, 98,9\% afirmam ter seu deslocamento à escola de forma ativa. Já entre os estudantes da escola particular, situada no centro da cidade, ape- 
nas $24 \%$ deslocam-se ativamente à escola, tendo a maioria destes (76\%) um deslocamento passivo. ${ }^{5}$ Os alunos que frequentam escolas públicas são mais propensos a se deslocarem ativamente do que os alunos que frequentam escolas particulares e uma das possíveis explicações para isso, é a diferença da distância do trajeto para a escola e/ou diferenças de posse de carro, que está diretamente relacionado com o nível socioeconômico. ${ }^{14}$

Pode-se concluir que, a maioria dos escolares avaliados se desloca até a escola de forma ativa. Os estudantes mais velhos, os meninos, os estudantes de escolas públicas e os de condições econômicas menos favoráveis apresentaram maiores percentuais de deslocamento ativo à escola.

É importante destacar que há poucas publicações a nível nacional avaliando deslocamento para a escola, o que dificultou a comparação com estudos relacionados com o deslocamento ativo de escolares. Fica assim evidente a necessidade de que outros estudos sejam realizados no Brasil, para a contribuição de futuras intervenções. Algumas variáveis como segurança da rota, aspectos ambientais e conhecimento dos benefícios da prática da atividade física ainda são pouco exploradas e talvez necessitem de um olhar diferenciado das autoridades públicas e da sociedade em geral para que melhores condições sejam oferecidas em favor da promoção da saúde e da qualidade de vida.

\section{REFERÊNCIAS}

1. Fonseca VM, Sichieri R, Veiga GV. Fatores associados à obesidade em adolescentes. Revista de Saúde Pública. 1998;32:541-549.

2. American College of Sports Medicine (ACSM). Diretrizes do ACSM para os testes de esforço e sua prescrição. 8. ed. Rio de Janeiro: Guanabara Koogan, 2012.

3. United States Department of Health and Human Services (USDHHS). Physical activity guidelines for Americans, Washington, 2008. [citado 2012 out 02] Disponível em: http://www. health.gov/paguidelines/pdf/paguide.pdf

4. Santos CM, Wanderley Júnior RS, Barros SSH, Farias Júnior JC, Barros MVG. Prevalência e fatores associados à inatividade física nos deslocamentos para escola em adolescentes. Cadernos de Saúde Pública. 2010; 26: 1419-1430.

5. Borfe L, Reuter CP, Meinhardt FP, Burgos MS. Atividades realizadas no tempo livre e tipo de deslocamento para a escola: um estudo com escolares do município de Santa Cruz do Sul, RS. EFDeportes.com, Revista Digital. 2010;16(163).

6. Rech RR, Costanzi CB, Bergmann MLA, Alli LR, Mattos AP, Trentin L et al. Prevalência de obesidade em escolares de 7 a 12 anos de uma cidade Serrana do RS, Brasil. Rev Bras Cineantropom Desempenho Hum 2010;12(2):90-97.

7. Barros AJD, Victora CG. Indicador econômico para o Brasil baseado no censo demográfico de 2000. Rev Saúde Pública 2005;39(4):523-529.

8. Skoffer B, Foldspang A. Physical activity and low-back pain in schoolchildren. European Spine Journal. 2008;17(3):373-9.

9. Chillón P, Ortega FB, Ruiz JR, Pérez IJ, Martín-Matillas M, Valtueña J et al. Socio-economic factors and active commuting to school in urban Spanish adolescents: the AVENA study. European Journal of Public Health. 2009;19(5):470-6.

10. Murtagh EM, Murphy MH.Active travel to school and physical activity levels of Irish primary schoolchildren. Pediatric Exercise Science. 2011;23(2):230-236.

11. Silva KS, Lopes AS. Excesso de peso, pressão arterial e atividade física no deslocamento à escola. Arquivos Brasileiros de Cardiologia. 2008;91(2):93-101.

12. Hallal PC,Bertoldi AD, Gonçalves H,Victora CG. Prevalência de sedentarismo e fatores associados em adolescentes de 10-12 anos de idade. Caderno de Saúde Pública 2006;22(6):1277-1287.

13. Sousa TF. Deslocamento passivo à escola em estudantes de um colégio particular da cidade de Itabuna, Bahia. EFDeportes.com, Revista Digital. 2010;15(146).

14. Davison KK, Werder JL, Lawson CT. Children's active commuting to school: current knowledge and future directions. Preventing Chronic Disease. 2008;5(3). 
15. McMillan TE. The relative influence of urban form on a child's travel mode to school. Transportation Research Part A. 2007;41(1):69-79.

16. Tammelin T, Ekelund U, Remes J, Näyhä S. Physical activity and sedentary behaviors among Finnish youth. Medicine \& Science in Sports \& Exercise. 2007;39(7):1067-74.

17. Baruki SBS. Estado Nutricional e Atividade Física em Escolares de 7 a 10 anos da rede Municipal de Ensino de Corumbá. Minas Gerais, 2004. [citado 2012 out 02] Disponível em: http://alexandria.cpd.ufv.br:8000/teses/ciencia\%20da\%20nutricao/2004/181307f.pdf

18. Silva DAS, Lima JO, Silva RJS, Prado RL. Nível de atividade física e comportamento sedentário em escolares. Rev Bras Cineantropom Desempenho Hum. 2009;11(3):299-306.

19. Wong BY, Faulker G, Buliung R, Irving H. Mode shifting in school travel mode: examining the prevalence and correlates of active school transport in Ontario, Canada. BMC Public Health. 2011;11:618.

20. Pabayo R, Gauvin L, Barnett TA. Longitudinal changes in active transportation to school in Canadian youth aged 6 through 16 years. Pediatrics. 2011;128(2):404-13.

21. Larsen K, Gilliland J, Hess P, Tucker P, Irwin J, He M. Identifying influences of physical environments and socio-demographic characteristics on a child's mode of travel to and from school. American Journal of Public Health. 2009;99(3):520-526.

Endereço para Correspondência Ricardo Rodrigo Rech. Endereço: Ivo Remo Comandulli, 160/31B - Caxias do

Recebido 29/05/2013

Revisado 17/08/2013

Sul, RS. ricardo.rech@gmail.com. 